\section{Silicone líquido industrial para transformar o corpo: prevalência e fatores associados ao seu uso entre travestis e mulheres transexuais em São Paulo, Brasil}

\author{
Use of industrial liquid silicone to transform the \\ body: prevalence and factors associated with \\ its use among transvestites and transsexual \\ women in São Paulo, Brazil
}

\author{
Silicona líquida industrial para transformar el \\ cuerpo: prevalencia y factores asociados a su \\ uso entre travestis y mujeres transexuales \\ en São Paulo, Brasil
}

Thiago Pestana Pinto 1

Flavia do Bonsucesso Teixeira 2

Claudia Renata dos Santos Barros 3

Ricardo Barbosa Martins 4

Gustavo Santa Roza Saggese 1

Daniel Dutra de Barros 1

Maria Amelia de Sousa Mascena Veras 1

doi: 10.1590/0102-311X00113316

\begin{abstract}
Resumo
O objetivo deste trabalho foi estimar a prevalência do uso de silicone líquido industrial (SLI) entre pessoas travestis e mulheres transexuais e identificar os fatores relacionados a esta prática. Trata-se de estudo transversal realizado em sete municípios do Estado de São Paulo, Brasil, com dados coletados entre 2014 e 2015, em uma amostra de 576 pessoas. Na análise dos fatores associados, utilizamos o modelo de Poisson com variância robusta para estimar as razões de prevalências bruta e ajustada. A prevalência do uso de SLI foi de 49\%, a média de idade para a primeira colocação de SLI foi de $22( \pm 5,3)$ anos

\section{Correspondência}

T. P. Pinto

Rua Sete de Abril 425, apto. 602, São Paulo, SP 01043-000,

Brasil.

thiago_pestana@yahoo.com.br

1 Faculdade de Ciências Médicas da Santa Casa de São Paulo, São Paulo, Brasil.

2 Universidade Federal de Uberlândia, Uberlândia, Brasil.

3 Universidade Católica de Santos, Santos, Brasil.

${ }_{4}$ Centro de Referência e Treinamento DST/AIDS-SP, São Paulo, Brasil.
\end{abstract} e aproximadamente $43 \%$ informaram a ocorrência de problemas de saúde decorrente do uso. No modelo múltiplo ter escolaridade menor que o nível superior, estar em faixa etária a partir dos 20 anos, identificar-se como travesti e exercer a prostituição foram associados positivamente com a utilização de SLI. Houve uma elevada prevalência do uso de SLI e de problemas decorrentes desta prática, indicando um desafio acerca da prevenção do uso e da redução dos danos à saúde provocados pelo SLI. Dessa forma, torna-se fundamental assegurar o acesso aos recursos necessários para a realização das modificações corporais ao longo do percurso de transição por meio de uma atenção integral à saúde das pessoas travestis e transexuais no Sistema Único de Saúde. Finalmente, incluir nas politicas de saúde as demandas por modificações corporais como parte da construção da identidade de gênero, respeitando as necessidades singulares de cada pessoa neste processo de transição.

Travestismo; Pessoas Transgênero; Aparência Física; Silicones 


\section{Introdução}

Travestis e transexuais sofrem um contínuo processo de exclusão e marginalização social, que resultam em desfechos mais desfavoráveis de saúde quando comparados com a população em geral, tais como altas taxas de infecção pelo HIV, experiência de violência, ideação suicida, entre outros 1,2,3. Apesar disso, em quase todos os países, incluindo o Brasil, são escassos os dados a respeito da situação de saúde e do impacto do processo de exclusão e de marginalização social sobre os vários aspectos da vida dessas pessoas 1 .

Para fins deste trabalho, ao nos referirmos às mulheres transexuais estamos recortando parte de um universo das transexualidades que não é homogêneo, mas aqui será representado por pessoas que tiveram seu gênero atribuído com base na genitália masculina e se reconhecem/reivindicam na posição de mulher 4 .

Da mesma forma, travestis são pessoas que se encontram nas fronteiras do gênero. E sem reduzi-las a uma única expressão, consideramos travestis aquelas que, também tendo sido atribuído pertencimento ao gênero masculino, transitam no universo feminino reivindicando esta identidade sem, no entanto, reivindicar a posição de mulher 5 .

Todavia, ressaltamos que essas categorias identitárias, além de serem permeadas por processos históricos e políticos na sua construção e definição, são fluidas e não dão conta da subjetividade e da diversidade de expressão de gênero entre essa população 6 .

Travestis e mulheres transexuais buscando uma "adequação" do corpo à identidade de gênero por meio da feminilização da aparência corporal podem utilizar diversos recursos ao longo do processo de transição, tais como hormônios, cirurgias plásticas, depilação a laser, uso de silicone líquido industrial (SLI) e outros 7,8 .

Com o objetivo de estabelecer critérios para o acompanhamento de pessoas transexuais e principalmente regulamentar a cirurgia de transgenitalização no Sistema Único de Saúde (SUS), o Ministério da Saúde, em 2008, institucionalizou o Processo Transexualizador no SUS por meio da Portaria GM/MS no 1.707, que não contemplava as travestis 9 . Em 2013, com o intuito de oferecer um cuidado integral à população de transexuais e travestis, a Portaria de 2008 foi revogada e substituída pela Portaria GM/MS no 2.803, que redefiniu e ampliou os cuidados incluídos, estabelecendo procedimentos, medicamentos, órteses, próteses e materiais a serem oferecidos pelo SUS, a exemplo do acompanhamento clínico, tratamento hormonal, cirurgia de transgenitalização e plástica mamária reconstrutiva com colocação de prótese de silicone 10 .

No entanto, em decorrência de obstáculos estruturais no acesso e de oferta insuficiente no SUS, seja em quantidade ou variedade de recursos e procedimentos, concomitantemente à exigência de condições e/ou critérios que não contemplam distintas necessidades, e considerando que as diversas pessoas travestis e transexuais desejam diferentes intervenções no decorrer do processo de transição, muitos procedimentos de mudança corporal contraindicados pela medicina, ou mesmo alguns dos que fazem parte do pacote regulamentado, continuam a ser realizados fora dos serviços de saúde por pessoas não habilitadas a despeito da regulamentação do Processo Transexualizador.

Dentre os procedimentos que acontecem fora dos serviços de saúde, destacaremos no presente estudo as injeções de SLI que são utilizadas por travestis e mulheres transexuais e que podem resultar em diversos problemas de saúde, tais como infecções, migração do produto para outras áreas do corpo, deformidades, siliconomas, necroses teciduais, embolia pulmonar e até a morte 11,12,13,14.

Acredita-se que desde a década de 1980 as injeções de SLI são usadas na modificação dos contornos corporais no Brasil 8,15,16. Entretanto, há que se diferenciar o SLI do chamado silicone cirúrgico, um produto purificado e estéril, que vem em embalagens especiais e foi desenvolvido para implantes em humanos. O SLI não é estéril e não tem indicação para ser aplicado em pessoas. Dentre os seus usos estão a lubrificação de máquinas, a lustração de painéis e de rodas de automóveis, e a vedação na construção civil. Em geral, as embalagens desse produto contêm advertências explícitas aos cuidados que se deve ter ao utilizá-lo, tais como evitar contato com a pele e inalação, devendo sua aplicação ocorrer somente em locais ventilados pelo risco de intoxicação.

Há relatos nas literaturas nacional e internacional da aplicação de outras substâncias consideradas inadequadas ao preenchimento corporal, sendo citadas parafina líquida, vaselina, óleo de linhaça, óleo de oliva, selante de pneus e fluido de transmissão automotivo 15,17,18. 
Além dos motivos relacionados ao Processo Transexualizador que podem contribuir para a continuação do uso do SLI, ressaltamos também os custos dos procedimentos que cumpririam os mesmos objetivos, como a colocação de próteses de silicone, bioplastia e lipoescultura pela rede privada de saúde, tornando as injeções de SLI a única opção viável para diversas travestis e transexuais que buscam transformações corporais. Por exemplo, em 2012 o valor médio para a colocação de próteses de silicone na região glútea nos Estados Unidos foi de US\$ 4.670, e os valores para aplicação de SLI variaram entre US\$ 300 e US\$ 1.600 18. No Brasil, em um estudo etnográfico realizado com travestis em Salvador, Bahia, houve menção de que em 1996 um litro de SLI custaria cerca de R\$ 200 16. Porém, hoje é possível comprar o produto pela internet ou em lojas especializadas por valores bem menores 19 .

No Brasil, apesar de ser essa uma prática sabidamente muito frequente, seu caráter clandestino é restritivo ao desenvolvimento de pesquisas que ampliem o conhecimento acerca das motivações para o uso do SLI, de suas consequências para a saúde e de outros fatores que possam estar a ele associados. A realização de estudos sobre o tema é necessária para que se possa verificar a magnitude desse problema de saúde e fornecer informações para a construção de estratégias e de políticas públicas mais eficientes.

Considerando o contexto, o objetivo deste trabalho foi estimar a prevalência da utilização do SLI e identificar os fatores associados a esta prática em uma amostra de travestis e mulheres transexuais residentes no Estado de São Paulo.

\section{Métodos}

Este trabalho analisa dados do Projeto Muriel: Vulnerabilidades, Demandas de Saúde e Acesso a Serviços da População de Travestis e Transexuais do Estado de São Paulo (Projeto Muriel). O projeto estudou uma amostra de 673 travestis e mulheres e homens transexuais em sete municípios do Estado de São Paulo (São Paulo, Campinas, São Bernardo do Campo, Santo André, Santos, São José do Rio Preto e Piracicaba) e combinou metodologia quantitativa e qualitativa.

Trata-se de um estudo transversal, com dados coletados no período entre 2014 e 2015 . A seleção amostral combinou uma amostra consecutiva, por cotas, proporcional ao tamanho, de pessoas travestis e transexuais que frequentam ou são acessadas pelos serviços de saúde e de assistência social dos municípios, complementada com a técnica "bola de neve", que tem como base a rede de relações sociais dos indivíduos pesquisados 20. Para estabelecer as cotas, foi realizado no ano anterior ao início do estudo um levantamento sobre o total de pessoas pertencentes a esses segmentos atendidas em atividades internas da unidade/serviço, bem como o número de pessoas que eram acessadas por agentes de saúde em atividades realizadas na rua, visto não dispormos de dados sobre o tamanho dessa população e sua localização espacial.

Foram adotados os seguintes critérios de inclusão: ter 16 anos ou mais na ocasião da entrevista; identificar-se como travesti, transexual ou transgênero; e residir no Estado de São Paulo há pelo menos seis meses.

A subamostra aqui analisada foi composta por 576 travestis e mulheres transexuais que responderam sobre o uso de SLI.

A variável dependente foi o uso do SLI, analisada pela pergunta: "Você já fez uso de silicone industrial?” (sim ou não). As variáveis independentes foram: faixa etária em anos (até 19, 20-29, 30-39, 40-49 e 50 ou +), raça/cor (branca, parda, preta e outra), Unidade Federativa de nascimento (UF), escolaridade (até o Ensino Fundamental incompleto, Ensino Fundamental completo, Ensino Médio completo e Ensino Superior completo), renda individual mensal (menor que um salário mínimo/ maior ou igual a um salário mínimo), moradia própria (sim ou não), trabalha com carteira assinada (sim ou não), tem convênio de saúde (sim ou não), como a pessoa se identifica/identidade de gênero (travesti ou transexual/transgênero), desejo de realizar cirurgia de transgenitalização (sim ou não), uso de hormônio sem receita médica (sim ou não) e faz programa (sim ou não).

As variáveis qualitativas foram descritas por meio de frequências absoluta e relativa e as variáveis numéricas valendo-se de médias e desvios padrão. Para analisar a hipótese de diferença entre as proporções foi utilizado o teste do qui-quadrado de Pearson ou exato de Fisher. A regressão de Poisson com variância robusta foi usada para analisar os fatores associados e estimar as razões de prevalência 
(RP), uma vez que a prevalência do desfecho foi alta 21,22. A análise múltipla foi construída baseando-se em um conjunto de variáveis que na literatura estão associadas com o uso de SLI ou que na análise univariada apresentaram um valor de $\mathrm{p}<0,20$. A entrada das variáveis no modelo foi feita de acordo com a ordem decrescente do valor de p. O ajuste do modelo final foi verificado por meio do goodness-of-fit-test e do linktest. Os dados foram analisados usando-se o pacote estatístico Stata 13 (StataCorp LP, College Station, Estados Unidos), com nível de significância adotado de 5\%.

O Projeto Muriel foi aprovado pelos Comitês de Ética em Pesquisa do Centro de Referência e Treinamento em DST/AIDS-SP (CRT DST/AIDS-SP) e da Secretaria Municipal de Saúde de São Paulo sob o registro (CAAE: 14277413.1.0000.53750) e está em consonância com as diretrizes para pesquisas em seres humanos no Brasil. Todas as participantes assinaram um Termo de Consentimento Livre e Esclarecido (TCLE) antes da condução das entrevistas.

\section{Resultados}

A amostra final foi de 576 pessoas, dentre as quais 283 se autoidentificaram como travestis e 293 como mulheres transexuais. A média de idade das participantes foi $32( \pm 9,72)$ anos, $43 \%$ delas estavam concentradas na faixa etária de 20 a 29 anos; a maioria (60\%) se declarou preta ou parda e 40\% dessas pessoas informaram não possuir renda ou referiram uma renda mensal individual menor que um salário mínimo. Em relação ao estado conjugal, a maioria (60\%) referiu estar sozinha/solteira. Quanto à escolaridade, aproximadamente 54\% das pessoas declararam ter completado no máximo o ensino fundamental e apenas 4,5\% completaram o ensino superior. Pouco mais da metade das pessoas entrevistadas nasceram no Estado de São Paulo (Tabela 1).

Quando perguntadas se tinham o nome social no Cartão Nacional de Saúde (CNS), cerca de duas em cada cinco pessoas referiram ter o nome civil no CNS. A maior parte das participantes dependia dos serviços de saúde ofertados pelo SUS, pois somente 14\% informaram ter convênio de saúde (Tabela 1).

Do total de participantes incluídas nesta análise, aproximadamente 95\% já fizeram uso de algum procedimento ou recurso com o intuito de modificar o corpo, e $96 \%$ utilizavam ou já haviam utilizado hormônio ao longo da vida (Tabela 2).

De cada cinco pessoas, pelo menos uma referiu ter sido discriminada nos serviços de saúde no último ano por ser travesti ou transexual. Ao longo da vida, essa proporção chegou a 42\%. Quase uma em cada três pessoas relatou já ter sofrido violência sexual, cerca de $48 \%$ já foram vítimas de violência policial e quase uma em cada quatro dessas pessoas informou já ter sido presa pelo menos uma vez (Tabela 2).

A utilização do SLI foi relatada por aproximadamente 49\% (IC95\%: 45,4-53,6) das entrevistadas. A média de idade para a primeira colocação de SLI foi de $22( \pm 5,3)$ anos, sendo a idade mínima 12 e a máxima 45 anos. Das 285 pessoas que colocaram o SLI, um total de 58 (20,42\%) realizou a primeira aplicação antes dos 18 anos de idade. Mais da metade $(51,41 \%)$ injetaram o produto pela primeira vez entre 20 e 29 anos (Tabela 2).

Dentre as pessoas que utilizaram o SLI, 121 (42\%) relataram ter tido problemas em decorrência desta prática. Quando questionadas sobre o que fizeram diante dos problemas apresentados, menos da metade $(46,25 \%)$ relatou ter procurado algum serviço público ou privado de saúde.

Mais da metade (52\%) das participantes que sofreram algum problema de saúde ou que tiveram sequelas decorrentes da utilização do SLI declararam-se satisfeitas, muito satisfeitas ou completamente satisfeitas com o resultado de sua aplicação.

Conforme o exposto na Tabela 3, por meio da análise bivariada observa-se que foram associadas positivamente ao uso de SLI as seguintes variáveis: estar na faixa etária de 30 a 49 anos, ter nascido fora do Estado de São Paulo, ter escolaridade inferior ao ensino superior, não ter moradia própria, não ter trabalho com carteira assinada, não ter convênio de saúde, identificar-se como travesti, não desejar fazer cirurgia de transgenitalização e fazer programa.

$\mathrm{Na}$ análise múltipla permaneceram associadas positivamente ao uso de SLI ter idade acima de 20 anos, possuir escolaridade menor que o nível superior, ter se identificado como travesti e exercer a prostituição (Tabela 4). 


\section{Tabela 1}

Descrição da amostra de pessoas travestis e mulheres transexuais conforme características sociodemográficas. Estado de São Paulo, Brasil, 2015 ( $n=576)$.

\begin{tabular}{|c|c|c|}
\hline Características & $\mathbf{n}$ & $\%$ \\
\hline \multicolumn{3}{|l|}{ Faixa etária em anos ( $n=576$ ) } \\
\hline $16-19$ & 24 & 4,17 \\
\hline $20-29$ & 248 & 43,06 \\
\hline $30-39$ & 169 & 29,34 \\
\hline $40-49$ & 110 & 19,10 \\
\hline $50 \mathrm{ou}+$ & 25 & 4,34 \\
\hline \multicolumn{3}{|l|}{ Raça/Cor $(n=574)$} \\
\hline Branca & 206 & 35,89 \\
\hline Parda & 256 & 44,60 \\
\hline Preta & 88 & 15,33 \\
\hline Outra & 24 & 4,18 \\
\hline \multicolumn{3}{|l|}{ Local de nascimento $(n=570)$} \\
\hline Estado de São Paulo & 296 & 51,92 \\
\hline Outro estado & 274 & 48,08 \\
\hline \multicolumn{3}{|l|}{ Estado marital $(n=574)$} \\
\hline Casada ou vivendo junto & 93 & 16,20 \\
\hline Namorando ou ficando & 93 & 16,20 \\
\hline Separada ou divorciada & 29 & 5,05 \\
\hline Sozinha ou solteira & 350 & 60,98 \\
\hline Viúva & 9 & 1,57 \\
\hline \multicolumn{3}{|l|}{ Escolaridade $(n=575)$} \\
\hline Até o Ensino Fundamental incompleto & 150 & 26,09 \\
\hline Ensino Fundamental & 164 & 28,52 \\
\hline Ensino Médio & 235 & 40,87 \\
\hline Ensino Superior & 26 & 4,52 \\
\hline \multicolumn{3}{|c|}{ Renda individual mensal em salários mínimos ( $n=541$ ) } \\
\hline$<1$ & 220 & 40,67 \\
\hline$\geq 1$ & 321 & 59,33 \\
\hline \multicolumn{3}{|l|}{ Trabalha com carteira assinada $(n=576)$} \\
\hline Não & 525 & 91,15 \\
\hline $\operatorname{Sim}$ & 51 & 8,85 \\
\hline \multicolumn{3}{|l|}{ Possui nome social no Cartão SUS ( $n=575$ ) } \\
\hline Não tem Cartão SUS & 55 & 9,60 \\
\hline Não possui o nome social & 244 & 42,40 \\
\hline Possui o nome social & 276 & 48,00 \\
\hline \multicolumn{3}{|l|}{ Tem convênio de saúde $(n=574)$} \\
\hline Não & 493 & 85,89 \\
\hline Sim & 81 & 14,11 \\
\hline
\end{tabular}

\section{Discussão}

Nossos dados apresentaram uma alta prevalência do uso de SLI entre as travestis e mulheres transexuais participantes do estudo, com quase metade das participantes relatando ter feito o procedimento. As pessoas entrevistadas se submeteram às aplicações de SLI ainda muito jovens e a frequência é maior entre aquelas com escolaridade mais baixa e que se autoidentificaram como travestis.

Apesar da relativa escassez de literatura científica sobre o tema, dados existentes revelam que essa prática é realizada com muita frequência em diversas partes do mundo. A prevalência aqui encontrada 


\section{Tabela 2}

Descrição da amostra de pessoas travestis e mulheres transexuais conforme características do percurso de transição, saúde, discriminação e violência. Estado de São Paulo, Brasil, $2015(n=576)$.

\begin{tabular}{|c|c|c|}
\hline Características & $\mathrm{n}$ & $\%$ \\
\hline \multicolumn{3}{|c|}{ Fez uso de recurso/procedimento para modificar o corpo $(n=575)$} \\
\hline Não & 25 & 7,35 \\
\hline Sim & 550 & 95,65 \\
\hline \multicolumn{3}{|c|}{ Já fez uso de silicone líquido industrial $(n=576)$} \\
\hline Não & 291 & 50,52 \\
\hline Sim & 285 & 49,48 \\
\hline \multicolumn{3}{|c|}{ Problemas decorrentes do silicone industrial $(n=285)$} \\
\hline Não & 164 & 57,55 \\
\hline $\operatorname{Sim}$ & 121 & 42,45 \\
\hline \multicolumn{3}{|c|}{ Já utilizou/utiliza hormônio ( $n=574)$} \\
\hline Não & 23 & 4,01 \\
\hline Sim & 551 & 95,99 \\
\hline \multicolumn{3}{|c|}{ Deseja realizar cirurgia de redesignação sexual $(n=571)$} \\
\hline Não & 428 & 74,96 \\
\hline Sim & 143 & 25,04 \\
\hline \multicolumn{3}{|c|}{ Sofreu discriminação por ser travesti ou transexual $(n=568)$} \\
\hline Não & 69 & 12,15 \\
\hline $\operatorname{Sim}$ & 499 & 87,85 \\
\hline \multicolumn{3}{|c|}{ Sofreu discriminação em serviços de saúde por ser travesti ou transexual $(n=576)$} \\
\hline Não & 330 & 57,29 \\
\hline $\operatorname{Sim}$ & 246 & 42,71 \\
\hline \multicolumn{3}{|c|}{ Sofreu discriminação em serviços de saúde por ser travesti ou transexual no último ano ( $n=576$ ) } \\
\hline Não & 453 & 78,65 \\
\hline Sim & 123 & 21,35 \\
\hline \multicolumn{3}{|c|}{ Já foi vítima de violência sexual $(n=576)$} \\
\hline Não & 392 & 68,06 \\
\hline Sim & 184 & 31,94 \\
\hline \multicolumn{3}{|c|}{ Já foi vítima de violência policial $(n=576)$} \\
\hline Não & 298 & 51,74 \\
\hline Sim & 278 & 48,26 \\
\hline
\end{tabular}

é mais baixa do que a relatada em um estudo realizado na Argentina em 2013, com uma amostra de 450 mulheres transexuais em que 61,6\% das participantes tinham usado SLI 23 . Na Tailândia, em estudo realizado em 2005, também foi observada uma alta prevalência $(68,6 \%)$ de implantes de próteses de silicone e de SLI em uma amostra de 325 mulheres transexuais. Todavia, não foi possível distinguir nesse estudo cirurgias para implante de próteses de silicone da prática de injeções de SLI 24.

Outras pesquisas encontraram prevalências menores do que a observada na presente investigação. Entre elas, uma foi realizada nos Estados Unidos, na cidade de Chicago em 2003, e mostrou que 29\% das pessoas em uma amostra por conveniência de 51 mulheres transexuais com idades entre 16 e 25 anos já teriam injetado SLI 25. Entre 1994 e 1995, foi realizado outro estudo por amostragem de conveniência com mulheres transexuais que faziam programa nas ruas de Roterdã, na Holanda, sendo o uso de SLI referido por cerca de 25\% das participantes 26. Também foi menor a prevalência encontrada em um estudo realizado numa casa de detenção na cidade de São Paulo no ano de 1990 com 82 travestis privadas de liberdade, sendo estimado o uso de SLI em 22\% 27.

Já um estudo realizado em 2013 em São Francisco, nos Estados Unidos 18, utilizando a metodologia respondent driven sampling (RDS) com uma amostra de 234 mulheres transexuais, encontrou uma 
Tabela 3

Fatores associados à utilização de silicone líquido industrial entre travestis e mulheres transexuais. Estado de São Paulo, Brasil, 2015.

\begin{tabular}{|c|c|c|c|c|c|}
\hline \multirow[t]{2}{*}{ Características } & \multicolumn{2}{|c|}{$\begin{array}{l}\text { Utilização de silicone } \\
\text { [n (\%)] }\end{array}$} & \multirow[t]{2}{*}{ RP (bruta) } & \multirow[t]{2}{*}{ IC95\% } & \multirow[t]{2}{*}{ Valor de $p$ * } \\
\hline & Não & Sim & & & \\
\hline Faixa etária em anos & & & & & $<0,001$ \\
\hline Até 19 & $22(91,70)$ & $2(8,30)$ & 1,00 & - & \\
\hline $20-29$ & $140(56,50)$ & $108(43,50)$ & 5,22 & $1,37-19,86$ & \\
\hline $30-39$ & $78(46,20)$ & $91(53,80)$ & 6,46 & $1,69-24,56$ & \\
\hline $40-49$ & $37(33,60)$ & $73(66,40)$ & 7,96 & $2,09-30,25$ & \\
\hline 50 ou + & $14(56,00)$ & $11(44,00)$ & 5,27 & $1,30-21,40$ & \\
\hline Raça/Cor & & & & & 0,142 \\
\hline Branca & $109(52,90)$ & $97(47,09)$ & 1,00 & - & \\
\hline Parda & $122(47,66)$ & $134(52,34)$ & 1,11 & $0,92-1,33$ & \\
\hline Preta & $47(53,41)$ & $41(46,59)$ & 0,98 & $0,75-1,29$ & \\
\hline Outra & $3(23,08)$ & $10(76,92)$ & 1,63 & $1,17-2,27$ & \\
\hline Local de nascimento & & & & & $<0,001$ \\
\hline Estado de São Paulo & $194(65,54)$ & $102(34,46)$ & 1,00 & - & \\
\hline Outro estado & $94(34,31)$ & $180(65,69)$ & 1,90 & $1,59-2,28$ & \\
\hline Escolaridade & & & & & $<0,001$ \\
\hline Ensino Superior completo & $22(84,62)$ & $4(15,38)$ & 1,00 & - & \\
\hline Ensino Médio completo & $121(51,49)$ & $114(48,51)$ & 3,15 & $1,26-7,84$ & \\
\hline Ensino Fundamental completo & $87(53,05)$ & $77(46,95)$ & 3,05 & $1,22-7,63$ & \\
\hline Até o Ensino Fundamental incompleto & $60(40,00)$ & $90(60,00)$ & 3,90 & $1,56-9,70$ & \\
\hline Renda individual mensal em salários mínimos ** & & & & & 0,074 \\
\hline$\geq 1$ & $150(46,70)$ & $171(53,30)$ & 1,00 & - & \\
\hline$<1$ & $120(54,50)$ & $100(45,50)$ & 0,85 & $0,71-1,01$ & \\
\hline Moradia própria & & & & & 0,002 \\
\hline Sim & $87(60,80)$ & $56(39,20)$ & 1,00 & - & \\
\hline Não & $161(45,70)$ & $191(54,30)$ & 1,38 & $1,10-1,73$ & \\
\hline Trabalha com carteira assinada & & & & & $<0,001$ \\
\hline Sim & $40(78,40)$ & $11(21,60)$ & 1,00 & - & \\
\hline Não & $251(47,80)$ & $274(52,20)$ & 2,41 & $1,42-4,11$ & \\
\hline Tem convênio de saúde & & & & & 0,008 \\
\hline Sim & $52(64,20)$ & $29(35,80)$ & 1,00 & - & \\
\hline Não & $238(48,30)$ & $255(51,70)$ & 1,44 & $1,06-1,95$ & \\
\hline Identidade de gênero (como identifica-se) & & & & & $<0,001$ \\
\hline Transexual & $175(59,93)$ & $117(40,07)$ & 1,00 & - & \\
\hline Travesti & $115(40,64)$ & $168(59,36)$ & 1,48 & $1,24-1,75$ & \\
\hline Deseja realizar cirurgia de redesignação sexual & & & & & 0,001 \\
\hline Sim & $89(62,24)$ & $54(37,76)$ & 1,00 & - & \\
\hline Não & $197(46,03)$ & $231(53,97)$ & 1,42 & $1,13-1,79$ & \\
\hline Histórico de uso de hormônio sem receita médica & & & & & 0,006 \\
\hline Não & $59(63,44)$ & $34(36,56)$ & 1,00 & - & \\
\hline Sim & $229(47,91)$ & $249(52,09)$ & 1,42 & $1,07-1,88$ & \\
\hline Faz programas & & & & & $<0,001$ \\
\hline Não & $193(59,57)$ & $131(40,43)$ & 1,00 & - & \\
\hline Sim & $97(39,11)$ & $151(60,89)$ & 1,50 & $1,27-1,77$ & \\
\hline
\end{tabular}

IC95\%: intervalo de 95\% de confiança; RP: razão de prevalência bruta.

* Teste do qui-quadrado;

** Salário mínimo no Estado de São Paulo à época da pesquisa = R\$905. 


\section{Tabela 4}

Modelo final da regressão de Poisson para fatores associados à utilização de silicone líquido industrial entre travestis e mulheres transexuais. Estado de São Paulo, Brasil, 2015

\begin{tabular}{|c|c|c|c|c|c|}
\hline Características & RP bruta & IC95\% & RP ajustada & IC95\% & Valor de $p$ * \\
\hline \multicolumn{6}{|l|}{ Faixa etária em anos } \\
\hline Até 19 & 1,00 & - & 1,00 & - & \\
\hline $20-29$ & 5,22 & $1,37-19,86$ & 5,08 & $1,39-18,47$ & 0,014 \\
\hline $30-39$ & 6,46 & $1,69-24,56$ & 7,35 & $2,02-26,64$ & 0,002 \\
\hline $40-49$ & 7,96 & $2,09-30,25$ & 8,64 & $2,38-31,30$ & 0,001 \\
\hline 50 ou + & 5,27 & $1,30-21,40$ & 6,50 & $1,67-25,21$ & 0,007 \\
\hline \multicolumn{6}{|l|}{ Escolaridade } \\
\hline Ensino Superior completo & 1,00 & - & 1,00 & - & \\
\hline Ensino Médio completo & 3,15 & $1,26-7,84$ & 3,24 & $1,33-7,89$ & 0,009 \\
\hline Ensino Fundamental completo & 3,05 & $1,22-7,63$ & 2,89 & $1,18-7,09$ & 0,020 \\
\hline Até o Ensino Fundamental incompleto & 3,90 & $1,56-9,70$ & 3,51 & $1,44-8,57$ & 0,006 \\
\hline \multicolumn{6}{|l|}{ Identidade de gênero (como identifica-se) } \\
\hline Transexual & 1,00 & - & 1,00 & - & \\
\hline Travesti & 1,48 & $1,24-1,75$ & 1,37 & $1,16-1,61$ & $<0,001$ \\
\hline \multicolumn{6}{|l|}{ Faz programas } \\
\hline Não & 1,00 & - & 1,00 & - & \\
\hline Sim & 1,50 & $1,27-1,77$ & 1,52 & $1,29-1,79$ & $<0,001$ \\
\hline
\end{tabular}

IC95\%: intervalo de 95\% de confiança; RP: razão de prevalência bruta.

* Regressão de Poisson com variância robusta.

prevalência muito menor ( $\mathrm{RP}=16,7 \%$; IC95\%: 9,9-25,2) para o uso do SLI e outras substâncias de preenchimento corporal quando comparado com a observada aqui.

Em relação à idade, foi observada no presente trabalho uma média de 22 anos na ocasião da primeira aplicação do SLI, sendo que mais da metade fizeram a primeira aplicação do produto entre 20 e 29 anos. Essa constatação levanta a possibilidade da utilização do SLI acontecer ao longo do processo de transição das travestis e mulheres transexuais, mais especificamente em momentos posteriores ao início do uso de hormônios, de vestimentas e adereços femininos e em períodos nos quais essas pessoas já dispunham de renda suficiente para pagar pela colocação do produto 7,18 . Se tomarmos como exemplo o caso das travestis de Salvador, a decisão de fazer uso de SLI inclui um período anterior de preparação, em que as candidatas precisam, além de conseguir o dinheiro para pagar, vencer o medo de realizar o procedimento 16.

Atualmente no Brasil, o Processo Transexualizador no SUS permite que travestis e mulheres transexuais iniciem o acompanhamento clínico a partir dos 18 anos, podendo realizar procedimentos cirúrgicos com a idade mínima de 21 anos e depois de já ter feito acompanhamento por tempo igual ou superior a dois anos 10 . Os nossos resultados mostram que uma em cada cinco pessoas que usaram SLI fez a primeira aplicação antes dos 18 anos, ou seja, antes da idade mínima para um possível ingresso no Processo Transexualizador. Isso mostra a importância de pensarmos estratégias e alternativas que contemplem as especificidades do intercurso do processo de transição para além do que já é previsto no atual modelo normatizado pela Portaria GM/MS no 2.803, compreendendo que em muitas ocasiões as intervenções corporais como uso de SLI ou a utilização de hormônios sem prescrição médica podem começar ainda na adolescência.

A prevalência de problemas decorrentes do SLI foi mais do que o dobro da encontrada pelo estudo realizado em São Francisco, em que 18\% relataram sua ocorrência (IC95\%: 5,3-40,2) 18. Contudo, no nosso trabalho mais da metade das pessoas que referiram problemas estão satisfeitas, muito satisfeitas ou completamente satisfeitas com os resultados das modificações corporais proporcionadas pelas injeções de silicone, já o estudo americano não dispunha desta informação. A satisfação com as trans- 
formações corporais resultantes das aplicações de SLI aqui observada, mesmo na frequente presença de problemas decorrentes deste procedimento, pode indicar que essas complicações sejam percebidas como inerentes ao processo de modificação corporal proporcionado por esta estratégia de risco. Porém, vale ressaltar que muitas vezes essa é a única alternativa para se obter determinadas transformações desejadas. A aplicação de SLI não pode ser analisada fora do contexto social em que ocorre, no qual o desejo e a necessidade da feminilização do corpo como parte da construção da identidade de gênero dessas pessoas se sobrepõem aos riscos do uso do SLI, mesmo quando estes são conhecidos 7.

Além disso, outro fator que pode explicar a satisfação e a repetição da prática de injetar o SLI, mesmo que se conheça os riscos a ele atribuídos, são os resultados imediatos desta intervenção, principalmente quando comparados ao uso de hormônios 15 . Vale destacar que seguir as recomendações e cuidados orientados pelas "bombadeiras" após o procedimento é considerado como fator importante para o alcance dos resultados esperados. Tais recomendações podem variar de acordo com a área do corpo e com a experiência de cada "bombadeira", mas incluem um período de repouso 7,8,16.

Apesar da alta prevalência de problemas relacionados ao uso do SLI, menos da metade dessas pessoas procurou por atendimento em serviços de saúde. Esse comportamento pode estar relacionado a fatores diversos, como o preconceito sofrido pelas travestis e mulheres transexuais em experiências anteriores nos serviços de saúde, assim como a falta de protocolos de atendimento e o desconhecimento dos profissionais de saúde para responder a tal demanda. Como observado em outros estudos, evitar a busca por cuidados em serviços de saúde pode constituir uma estratégia adotada por travestis e mulheres transexuais para contornar a violência sofrida nestes ambientes 28,29.

Como anteriormente mencionado, em geral as aplicações são realizadas por outras travestis ou mulheres transexuais mais experientes, conhecidas como "bombadeiras" 7,28, como podemos observar no estudo realizado na Argentina, em que 91,7\% tiveram o SLI injetado por outras mulheres transexuais 23 , bem como em dois estudos etnográficos realizados no Brasil 8,16. Estudos qualitativos mostram que em grande medida os cuidados relacionados ao uso do SLI também são orientados pelas próprias "bombadeiras" ou ocorrem por meio da automedicação 7,8,16,28.

Em nossa pesquisa não investigamos em que áreas do corpo se injeta o SLI mais frequentemente, mas um estudo nacional 16 refere a preferência por glúteos, coxas e quadris, ao contrário de trabalhos realizados nos Estados Unidos, Holanda e Tailândia que mostram que as partes do corpo mais modificadas por meio de SLI são as mamas e a face 18,24,26, o que pode significar que a adequação se inspira em modelos de beleza e sexualidade valorizados em cada um desses contextos. Outra consideração relevante é que, no Brasil, na medida em que as próteses de silicone para as mamas ficaram mais acessíveis, as aplicações de SLI nestas áreas do corpo podem estar diminuindo.

Entre as pessoas que não mencionaram o desejo de realizar a cirurgia de transgenitalização, o SLI foi usado por mais de $50 \%$. Esse achado nos permite questionar as etapas do cuidado proposto pelo chamado Processo Transexualizador no SUS, em que a colocação de próteses de silicone bilateral nas mamas só está prevista como procedimento complementar à cirurgia de transgenitalização ${ }^{10}$. Ou seja, foi demonstrado por este e por outros estudos que travestis ou mulheres transexuais que desejem realizar modificações corporais, tal como a implantação de próteses de silicone nas mamas, mas que não queiram a cirurgia de transgenitalização não terão acesso a tal procedimento por meio do SUS 30 . Assim, as normas do Processo Transexualizador obliteram o que pode ser uma das principais reivindicações das travestis referente às transformações corporais ofertadas pelo SUS. Em relação às mulheres transexuais, ainda que queiram realizar a cirurgia de transgenitalização, este condicionamento produz uma dupla violação, considerando-se as intermináveis filas de espera para realizar o procedimento cirúrgico e o seu acesso às próteses.

Dentre os recursos mais usados nas modificações corporais estão os hormônios. Na amostra estudada, travestis e mulheres transexuais com histórico de utilização de hormônio sem prescrição médica apresentaram maior prevalência de uso do SLI, em consonância com o encontrado no estudo realizado em São Francisco 18. Destacamos que o processo de transição parece ser marcado pelas práticas que estão à margem do sistema oficial de saúde, tais como a terapia hormonal por automedicação e o uso do SLI.

Entre as participantes que referiram fazer programas, observamos uma prevalência de uso de SLI 52\% maior em comparação ao grupo que não informou tal atividade. Uma pesquisa realizada na Argentina também encontrou maior prevalência da utilização de SLI em pessoas com histórico de 
trabalho sexual 23. Já no estudo feito em São Francisco, não houve associação entre o uso de SLI e o histórico de trabalho sexual. No entanto, considerando que a prostituição é crime nos Estados Unidos, é possível que essa informação possa ter sido omitida pelas participantes do estudo ou que de fato sua ocorrência seja muito menor na população pesquisada naquela cidade 18 .

Cabe salientar aqui que as pesquisas realizadas com travestis e mulheres transexuais, desde a década de 1990, registraram recorrentemente os espaços de prostituição como cenários para coletar dados ou acessar informantes. $\mathrm{O}$ fato de as travestis e mulheres transexuais trabalharem no mercado sexual não deve ser naturalizado, mas sim discutido em articulação com os contextos de acesso, de permanência no trabalho (incluindo a regulamentação do trabalho sexual) e de transfobia.

Como já mencionado, o uso do SLI pode proporcionar as transformações corporais desejadas com maior rapidez, representando uma valorização do capital corporal, o que significa para as pessoas que trabalham no mercado sexual um incremento no valor e no número de clientes 7,31. Por outro lado, algumas das pessoas que utilizam o SLI ou fazem outros procedimentos podem ter de elevar a carga de trabalho no mercado sexual para viabilizar o seu pagamento, aumentando riscos como a infecção pelo HIV, à medida em que têm piores condições para negociar com os clientes, tornando-as mais vulneráveis às práticas desprotegidas 32 .

Não é surpreendente que pessoas com escolaridade correspondente ao nível superior tenham apresentado uma prevalência acentuadamente menor de utilização do SLI em relação àquelas com menor escolaridade. No nosso trabalho, a prevalência foi 3,5 vezes maior no grupo de pessoas que tinham apenas até o Ensino Fundamental quando comparadas às participantes que informaram ter nível superior. Entre outros fatores, pessoas com menor escolaridade podem ter uma trajetória permeada por maior vulnerabilidade, piores oportunidades de trabalho e limitado acesso aos serviços de saúde, tornando-se mais propensas à utilização de estratégias precárias para a sua transformação corporal. Os estudos realizados na Argentina 23 e em São Francisco 18 não encontraram diferenças de uso do SLI entre os diversos níveis de escolaridade.

Em relação às categorias identitárias, as pessoas que se autoidentificaram como travestis apresentaram uma prevalência 37\% maior de uso do SLI quando comparadas àquelas que se identificaram como transexuais. No Brasil, a categoria identitária transexual é relativamente recente, sendo registrada somente a partir dos anos 2000, e a denominação travesti foi consolidada nos anos 1970 6. Como já exposto, essas categorias não abarcam a subjetividade da expressão de gênero entre as pessoas trans, assim como também podem variar ao longo do percurso de transição.

Todavia, no que concerne à categoria "travesti" ainda existe na sociedade um forte estigma, sendo a mesma recorrentemente associada à marginalização e ao mercado do sexo 33 . Leite 33 acrescenta que em relação ao termo "travesti”, o termo "transexual” possui um capital linguístico mais valorizado. Dessa forma, pessoas que se identificam como travestis podem vivenciar uma situação de maior vulnerabilidade e exclusão social, ficando mais expostas a alternativas precárias de procedimentos com o intuito de promover as modificações corporais. Vale ressaltar que o uso do SLI pode ser uma prática que traz tanto uma conotação de pertencimento ao grupo quanto ser estigmatizadora mesmo entre as travestis. Conforme aponta Pelúcio 7 (p. 103), "bombar-se é entrar definitivamente no mundo das travestis e com ele pactuar. Por isso, algumas travestis 'tops' asseguram que não têm nem nunca terão esse 'lixo' no corpo".

Observamos em alguns trabalhos que a maioria das pessoas que se identificam como travestis não deseja realizar a cirurgia de transgenitalização 7,8,33. Porém, destacamos que tal condição não é absoluta, ou seja, podemos encontrar pessoas que se autoidentificam como travestis e que em algum momento da vida possam desejar realizar a cirurgia de transgenitalização, assim como pessoas que se autoidentificam como transexuais sem a pretensão de realizar esta cirurgia. Esse achado é interessante porque dialoga com os estudos mais recentes que criticam a "identidade cirúrgica" atribuída às pessoas transexuais que reiteram a patologização de suas experiências 4,34,35.

Este estudo tem limitações, dentre as quais o fato de as participantes terem sido selecionadas em serviços de saúde e de assistência social, incluindo os serviços de atenção à saúde das pessoas trans, o que pode resultar em viés de seleção em relação a alguns desfechos, em especial os relacionados à saúde.

No entanto, apesar das limitações, acreditamos que este é o primeiro trabalho com uma amostra grande de pessoas trans a revelar importantes dados acerca da utilização do SLI nesta população no Brasil. Os resultados apresentam alta prevalência do uso de SLI, bem como de problemas de saúde 
decorrentes. Os seus achados evidenciam a necessidade urgente da ampliação de estratégias de atenção à saúde de travestis e mulheres transexuais, incluindo a revisão do Processo Transexualizador no SUS, de modo a diferenciar e oferecer os procedimentos de modificação corporal, seja terapia hormonal, cirurgias plásticas, bioplastia ou a colocação de próteses de silicone, não como um pacote único, mas adequados às necessidades de cada pessoa, reconhecendo a diversidade das demandas de saúde como parte da construção da identidade de gênero, assim como a singularidade com que cada indivíduo vivencia esse processo de transição, assegurando de fato uma atenção integral à saúde.

\section{Colaboradores}

T. P. Pinto contribuiu com a concepção do estudo, realização das análises e redação final. F. B. Teixeira e R. B. Martins contribuíram com a concepção do estudo e aprovaram a versão final. C. R. S. Barros colaborou com a análise estatística, coleta de dados e aprovação da versão final. G. S. R. Saggese contribuiu com a concepção do estudo, discussão e aprovação da versão final. D. D. Barros contribuiu com a concepção do estudo, coleta de dados e aprovação da versão final. M. A. S. M. Veras contribuiu com a concepção do estudo, supervisão das análises e aprovação da versão final.

\section{Agradecimentos}

Agradecemos à Fundação de Amparo à Pesquisa do Estado de São Paulo (Fapesp; processo 2013/223667) pelo financiamento do projeto, ao apoio do Centro de Referência e Treinamento DST/AIDS-SP e ao Grupo de Pesquisa Muriel.

\section{Referências}

1. United Nations Development Programme. Transgender Health and Human Rights. New York: United Nations Development Programme; 2013.

2. Secretaria de Direitos Humanos, Presidência da República. 2o relatório sobre violência homofóbica no Brasil. Brasília: Secretaria de Direitos Humanos, Presidência da República; 2012.

3. Baral SD, Poteat T, Strömdahl S, Wirtz AL, Guadamuz TE, Beyrer C. Worldwide burden of HIV in transgender women: a systematic review and meta-analysis. Lancet Infect Dis 2013; 13:214-22.

4. Teixeira FB. Dispositivos de dor: saberes - poderes que (con)formam as transexualidades. São Paulo: Editora Annablume; 2013.

5. Ribeiro DC, Teixeira FB. Não é apenas um nome: a luta por reconhecimento no universo trans. In: Cordeiro CJ, Gomes JA, organizadores. Temas contemporâneos de direito das famílias. v. 2. 2a Ed. São Paulo: Editora Pillares; 2015. p. 499-524.

6. Carvalho M, Carrara S. Em direito a um futuro trans? Contribuição para a história do movimento de travestis e transexuais no Brasil. Sex Salud Soc (Rio J.) 2013; 8:319-51.

7. Pelúcio L. "Toda quebrada na plástica": corporalidade e construção de gênero entre travestis paulistas. Campos: Revista de Antropologia Social 2005; 6:97-112.

8. Benedetti M. Toda feita - o corpo e o gênero das travestis. Rio de Janeiro: Editora: Garamond; 2005. (Coleção Sexualidade, Gênero e Sociedade).

9. Ministério da Saúde. Portaria no 1.707 , de 18 de agosto de 2008. Institui, no âmbito do Sistema Único de Saúde (SUS), o Processo Transexualizador, a ser implantado nas unidades federadas, respeitadas as competências das três esferas de gestão. Diário Oficial da União 2008; 19 ago. 
10. Ministério da Saúde. Portaria no 2.803, de 19 de novembro de 2013. Redefine e amplia o Processo Transexualizador no Sistema Único de Saúde. Diário Oficial da União 2013; 21 nov.

11. Narins RS, Beer K. Liquid injectable silicone: a review of its history, immunology, technical considerations, complications, and potential. Plast Reconstr Surg 2006; 118(3 Suppl): 77S-84S.

12. Ensina LF, Tanno LK, Rodrigues AT, Garro LS, Giavina-Bianchi P, Motta AA. Hemorragia alveolar após injeção parenteral de silicone industrial. Rev Port Imunoalergol 2009; 17:563-4.

13. Mello DF, Gonçalves KC, Fraga MF, Perin LF, Helene Jr. A. Complicações locais após a injeção de silicone líquido industrial: série de casos. Rev Col Bras Cir 2013; 40:37-43.

14. Arán M, Murta D, Lionço T. Transexualidade e saúde pública no Brasil. Ciênc Saúde Coletiva 2009; 14:1141-9.

15. Guimarães A. Todas as mulheres do mundo: a construção do corpo travesti no Brasil das décadas de 1960 e 1970. In: Ministério da Saúde, organizador. Transexualidade e travestilidade na saúde. Brasília: Ministério da Saúde; 2015. p. 39-63.

16. Kulick D. Travesti: prostituição, sexo, gênero e cultura no Brasil. Rio de Janeiro: Editora Fiocruz; 2008.

17. Styperek A, Bayers S, Beer M, Beer K. Nonmedical-grade injections of permanent fillers. J Clin Aesthet Dermatol 2013; 6:22-9.

18. Wilson E, Rapues J, Jin H, Raymond HF. The use and correlates of illicit silicone or "fillers" in a population-based sample of transwomen, San Francisco, 2013. J Sex Med 2014; 29: 997-1003.

19. Pereira MJA. "É a dor da beleza" - as travestis e suas corajosas. In: Anais Eletrônicos do Seminário Internacional Fazendo Gênero 10. http://www.fazendogenero.ufsc.br/10/re sources/anais/20/1386738567_ARQUIVO_ MarlysonJunioAlvarengaPereira.pdf (acessado em 25/Jun/2016).

20. Shaghaghi A, Bhopal RS, Sheikh A. Approaches to recruiting "hard-to-reach" populations into re-search: a review of the literature. Health Promot Perspect 2011; 1:86-94.

21. Barros AJD, Hirakata VN. Alternatives for logistic regression in cross-sectional studies: an empirical comparison of models that directly estimate the prevalence ratio. BMC Med Res Methodol 2003; 3:21.

22. Coutinho LMS, Scazufca M, Menezes PR. Methods for estimating prevalence ratios in cross-sectional studies. Rev Saúde Pública 2008; 42:992-8.

23. Socias ME, Kerr T, Marshall BDL, Arístegui I, Frola C, Pérez H, et al. Prevalence and correlates of injection of industrial silicone among transgender women in Argentina. In: 8th IAS Conference on HIV Pathogenesis. http://www. cfenet.ubc.ca/sites/default/files/uploads/ IAS2015/posters/TUPEC567-Socias-MariaEugenia.pdf (acessado em 24/Fev/2016).
24. Guadamuz TE, Wimonsate W, Varangrat A, Phanuphak P, Jommaroeng R, McNicholl JM, et al. HIV prevalence, risk behavior, hormone use and surgical history among transgender persons in Thailand. AIDS Behav 2011; 15:650-8.

25. Garofalo R, Deleon J, Osmer E, Doll M, Harper GW. Overlooked, misunderstood and at-risk: exploring the lives and HIV risk of ethnic minority male-to-female transgender youth. J Adolesc Health 2006. 38:230-6.

26. Wiessing L, van Roosmalen M, Koedijk P, Bieleman B, Houweling H. Silicones, hormones and HIV in transgender street prostitutes. AIDS 1999; 13:2315-6.

27. Varella L, Tuason M, Proffitt N, Escaleira A, Alquezar A, Bukowski R. HIV infection among Brazilian transvestite in prison population. AIDS Patient Care 1996; 10:299-303.

28. Souza MHT, Signorelli MC, Coviello DM, Pereira PPG. Itinerários terapêuticos de travestis da região central do Rio Grande do Sul, Brasil. Ciênc Saúde Coletiva 2014; 19:2277-86.

29. Souza MHT, Malvasi P, Signorelli MC, Pereira PPG. Violência e sofrimento social no itinerário de travestis de Santa Maria, Rio Grande do Sul, Brasil. Cad Saúde Pública 2015; 31:76776.

30. Teixeira FB. (Des)engano: revisando as portarias do Processo Transexualizador no SUS. In: Uziel AP, Guilhon F, organizadores. Transdiversidades: práticas e diálogos em trânsitos. Rio de Janeiro: Eduerj; 2017. p. 313-52.

31. Vasconcelos OS, Cal DG, Mokarzel MO. Tinha travesti brincando de "pira": construção simbólica de hierarquias e territorialidades na prática da prostituição. Revista Latino-americana de Geografia e Gênero 2016; 7:46-58.

32. Wallace PM. Finding self: a qualitative study of transgender, transitioning, and adulterated silicone. Health Educ J 2010; 69:439-46.

33. Leite Jr. J. "Nossos corpos também mudam": sexo, gênero e a invenção das categorias "travesti" e "transexual" no discurso científico [Tese de Doutorado]. São Paulo: Pontifícia Universidade Católica de São Paulo; 2008.

34. Borba R. O (des)aprendizado de si: transexualidades, interação e cuidado em saúde. Rio de Janeiro: Editora Fiocruz: 2016.

35. Bento B. A reinvenção do corpo: sexualidade e gênero na experiência transexual. 2a Ed. Natal: EDUFRN; 2014. 


\section{Abstract}

This study aimed to estimate the prevalence of use of industrial liquid silicone (ILS) among transvestite persons and transsexual women and identify associated factors. This was a cross-sectional study in seven municipalities in São Paulo State, Brazil, with data collected in 2014 and 2015 in a sample of 576 individuals. Analysis of the associated factors used a Poisson model with robust variance to estimate the crude and adjusted prevalence ratios. Prevalence of use of ILS was 49\%, mean age at first injection of ILS was $22( \pm 5.3)$ years, and $43 \%$ reported health problems resulting from its use. Having less than a university education, age 20 years and older, self-identification as transvestite, and sex work were positively associated with use of ILS according to the multivariate model. There was a high prevalence of ILS use and resulting health problems, indicating the need to prevent its use and reduce the resulting health problems. It is thus essential to ensure access to the necessary resources for body changes during transition through comprehensive care for transvestites and transsexual persons in the Brazilian Unified $\mathrm{Na}$ tional Health System (SUS). Finally, health policies should include demands for body changes as part of gender identity construction, respecting each person's unique needs in this transition process.

Transvestism; Transgender Persons; Physical Appearance; Silicones

\section{Resumen}

El objetivo de este trabajo fue estimar la prevalencia del uso de silicona líquida industrial (SLI) entre personas travestis y mujeres transexuales e identificar los factores relacionados con esta práctica. Se trata de un estudio transversal, realizado en siete municipios del estado de São Paulo, Brasil, con datos recogidos entre 2014 y 2015, en una muestra de 576 personas. En el análisis de los factores asociados, utilizamos el modelo de Poisson con variancia robusta para estimar las razones de prevalencias bruta y ajustada. La prevalencia del uso de SLI fue de un 49\%, la media de edad para la primera colocación de SLI fue de $22( \pm 5,3)$ años $y$, aproximadamente, un 43\% informaron la ocurrencia de problemas de salud derivada de su uso. En el modelo múltiple tener escolaridad inferior al nivel superior, estar en una franja de edad a partir de los 20 años, identificarse como travesti y ejercer la prostitución estuvieron asociados positivamente con la utilización de SLI. Hubo una elevada prevalencia del uso de SLI y de problemas derivados de esta práctica, indicando un desafío acerca de la prevención del uso y de la reducción de los daños a la salud provocados por el SLI. De esta forma, es perentorio asegurar el acceso a los recursos necesarios para la realización de las modificaciones corporales a lo largo de la duración de esta transición, mediante una atención integral a la salud de las personas travestis y transexuales en el Sistema Único de Salud. Finalmente, incluir en las politicas de salud las solicitudes de modificaciones corporales como una parte de la construcción de la identidad de género, respetando las necesidades singulares de cada persona en este proceso de transición.

Travestismo; Personas Transgénero; Apariencia Física; Silicones

Recebido em 29/Jun/2016

Versão final reapresentada em 18/Ago/2016

Aprovado em 08/Set/2016 